\title{
Drag force, diffusion coefficient, and electric mobility of small particles. II. Application
}

\author{
Zhigang Li and Hai Wang \\ Department of Mechanical Engineering, University of Delaware, Newark, Delaware 19716, USA
}

(Received 25 July 2003; published 31 December 2003)

\begin{abstract}
We propose a generalized treatment of the drag force of a spherical particle due to its motion in a laminar fluid media. The theory is equally applicable to analysis of particle diffusion and electric mobility. The focus of the current analysis is on the motion of spherical particles in low-density gases with Knudsen number Kn $\gg 1$. The treatment is based on the gas-kinetic theory analysis of drag force in the specular and diffuse scattering limits obtained in a preceding paper [Z. Li and H. Wang, Phys. Rev. E., 68, 061206 (2003)]. Our analysis considers the influence of van der Waals interactions on the momentum transfer upon collision of a gas molecule with the particle and expresses this influence in terms of an effective, reduced collision integral. This influence is shown to be significant for nanosized particles. In the present paper, the reduced collision integral values are obtained for specular and diffuse scattering, using a Lennard-Jones-type potential energy function suitable for the interactions of a gas molecule with a particle. An empirical formula for the momentum accommodation function, used to determine the effective, reduced collision integral, is obtained from available experimental data. The resulting treatment is shown to be accurate for interpreting the mobility experiments for particles as small as $\sim 1 \mathrm{~nm}$ in radius. The treatment is subsequently extended to the entire range of the Knudsen number, following a semiempirical, gas-kinetic theory analysis. We demonstrate that the proposed formula predicts very well Millikan's oil-droplet experiments [R. A. Millikan, Philos. Mag. 34, 1 (1917); Phys.

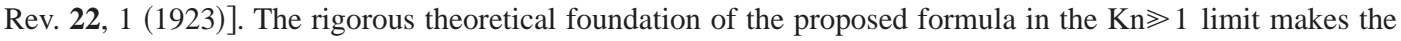
current theory far more general than the semiempirical Stokes-Cunningham formula in terms of the particle size and condition of the fluid and, therefore, more attractive than the Stokes-Cunningham formula.
\end{abstract}

DOI: 10.1103/PhysRevE.68.061207 PACS number(s): 47.45.Dt, 05.20.Dd, 05.60.Cd, 65.80. $+\mathrm{n}$

\section{INTRODUCTION}

The drag force on a small, spherical particle due to its motion in a laminar fluid has been historically given by the Stokes-Cunningham formula

$$
\mathbf{F}=-\frac{6 \pi \mu R \mathbf{V}}{1+\operatorname{Kn}[A+B \exp (-E / \mathrm{Kn})]},
$$

where $\mu$ is the viscosity of the gas, $R$ is the radius of the particle, $\mathbf{V}$ is the velocity vector of the particle relative to the gas, $\mathrm{Kn}$ is the Knudsen number defined as $\lambda / R, \lambda$ is the mean free path of the gas molecules, and $A, B$, and $E$ are empirical constants determined from the much celebrated, oil-droplet experiments of Millikan [1-3].

In the preceding paper [4] (hereafter referred to as paper I), we reviewed the current state of knowledge of the transport of small, spherical particles, including drag force, diffusion, and electric mobility [5], and concluded that a generalized theory of particle transport is not available. Specifically, we showed that in the free-molecule regime, Eq. (1) is valid only when the collision integral of fluid viscosity, $\Omega^{(2,2)^{*}}$, is around unity. As such, it is applicable only to particle motion in air at room temperature. Furthermore, we demonstrated that Eq. (1) is not applicable when a particle approaches the size of a molecule. The problem arises from the disagreement between Eq. (1) and the Chapman-Enskog theory of molecular diffusion $[6,7]$.

Using the gas-kinetic theory and considering the potential force of interactions during the collision of particle and fluid molecules, we obtained a rigorous and generalized treatment of momentum transfer in the free-molecule regime. More specifically, we examined the limiting cases of specular and diffuse scattering of fluid molecules upon collision with a spherical particle. The influence of potential force of interactions was cast into reduced collision integrals for the two limiting cases, following the same approach as the ChapmanEnskog theory $[6,7]$. The resulting drag force is given by

$$
\mathbf{F}_{\infty}=-\frac{8}{3} \sqrt{2 \pi m_{r} k T} N R^{2} \Omega_{a v g}^{(1,1) *} \mathbf{V},
$$

where the subscript $\infty$ denotes the free-molecule regime or $\mathrm{Kn} \gg 1, m_{r}=m_{g} m_{p} /\left(m_{g}+m_{p}\right)$ is the reduced mass, $m_{g}$ and $m_{p}$ are the masses of the gas molecule and particle, respectively, $k$ is the Boltzmann constant, $T$ is the absolute temperature, and $N$ is the number density of the gas. In Eq. (2), $\Omega_{a v g}^{(1,1)^{*}}$ is an effective, reduced collision integral. On the basis of Millikan's experimental evidence and the ChapmanEnskog theory, $\Omega_{a v g}^{(1,1)^{*}}$ must be bound by the limiting collision integrals of diffuse and specular scattering. In paper I, we proposed that $\Omega_{a v g}^{(1,1)^{*}}$ be modeled by

$$
\Omega_{a v g}^{(1,1)^{*}}=\varphi \Omega_{d}^{(1,1)^{*}}+(1-\varphi) \Omega_{s}^{(1,1)^{*}}
$$

where $\varphi(0 \leqslant \varphi \leqslant 1)$ is, in fact, the momentum accommodation coefficient [8], and $\Omega_{d}^{(1,1)^{*}}$ and $\Omega_{s}^{(1,1)^{*}}$ are the reduced collision integrals for diffuse and specular scattering, respectively. In paper I, we obtained mathematical expressions for $\Omega_{d}^{(1,1)^{*}}$ and $\Omega_{s}^{(1,1)^{*}}$. In the limit of rigid-body collisions, we found that $\Omega_{d}^{(1,1)^{*}}=1+\pi / 8$ and $\Omega_{s}^{(1,1)^{*}}=1$, and for nonrigid-body collisions the reduced collision integrals usually 


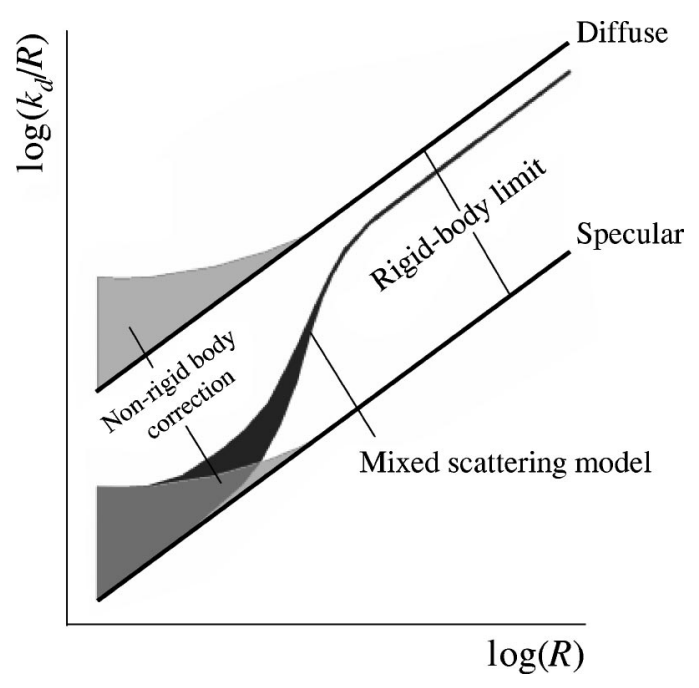

FIG. 1. Schematic of the drag coefficient as a function of particle radius for $\mathrm{Kn} \gg 1$. The bends above the hard-sphere limits are caused by the influence of the van der Waals force on the collision cross section. The mixed-scattering model shows the transition from specular scattering to diffuse scattering as the particle radius increases (see text).

assume values larger than those of the rigid-body limit. By comparing the Chapman-Enskog theory of molecular diffusion and Millikan's analysis of his data in the context of Epstein's [9] equations of drag force, we concluded in paper I that the momentum-accommodation factor is a function of particle size. Therefore, we shall hereafter call $\varphi$ the momentum-accommodation function.

Figure 1 depicts the schematic variation of the drag coefficient for $\mathrm{Kn} \gg 1, k_{d, \infty}=-\mathbf{F}_{\infty} / \mathbf{V}$, as a function of particle size in the limits of specular and diffuse scattering (thin lines). The shaded bands in Fig. 1 represent the correction due to non-rigid-sphere collisions. The mixed scattering model, shown by the dark band, accounts for the non-rigidsphere correction as well as the variation of $\varphi$ as a function of the particle size.

The particle transport theory given by Eqs. (2) and (3) contains several important features [4]. With $\varphi=0$ the theory reproduces the first approximation of the Chapman-Enskog theory of molecular diffusion. Second, Epstein's drag force formulations [9] are shown to be special cases of the current theory in the limit of rigid-body collisions. Last, the current theory should reproduce Millikan's oil-droplet data with a $\varphi$ value of about 0.9 . As such this theory is general with respect to the variation of particle size and the fluid conditions as long as $\mathrm{Kn} \gg 1$.

The objective of the current paper is to develop a simple mathematical model for the momentum-accommodation function $\varphi$ on the basis of available experimental data. The values of reduced collision integrals are tabulated for a Lennard-Jones-type potential energy function, as will be discussed in Sec. II. In Sec. III, we will parametrize the momentum-accommodation function and demonstrate that the theory given by Eqs. (2) and (3) is quantitatively predictive against existing experimental data. In Sec. IV, an extension of the current theory to the transition $(\mathrm{Kn} \sim 1)$ and con- tinuum $(\mathrm{Kn} \ll 1)$ regimes is developed following a semiempirical approach. The resulting generalized theory of spherical particle transport is shown to be applicable over the entire range of the Knudsen number.

\section{COLLISION INTEGRALS AND POTENTIAL ENERGY OF INTERACTIONS}

In paper I, the expression for the reduced collision integral was obtained as

$$
\Omega_{d(s)}^{(1,1) *}=\frac{\int_{0}^{\infty} \exp \left(-\gamma^{2}\right) \gamma^{5} Q_{d(s)}(g) d \gamma}{\pi R^{2}}
$$

where $Q_{d(s)}(g)$ is the energy-dependent collision cross section and $g=\gamma \sqrt{2 \mathrm{kT/m} / \mathrm{m}_{r}}$. Although our theoretical development differs from the approach of the Chapman-Enskog theory of molecular diffusion [4], Eq. (4) is identical to the first approximation of that theory. The expressions for the collision cross section were also developed. For diffuse scattering, we have

$$
\begin{aligned}
Q_{d}(g)= & 2 \pi\left[\int_{0}^{b_{0}}\left(1+\frac{1}{g} \sqrt{\frac{\pi k T}{2 m_{r}}} \sin \frac{\chi}{2}\right) b d b\right. \\
& \left.+\int_{b_{0}}^{\infty}(1-\cos \chi) b d b\right],
\end{aligned}
$$

and for specular scattering,

$$
Q_{s}(g)=2 \pi \int_{0}^{\infty}(1-\cos \chi) b d b,
$$

where $\chi$ is the scattering angle, $b$ is the impact parameter [7], and $b_{0}$ is the critical impact parameter below which the gas molecule undergoes physical contact with the particle in a scattering process $[4,7]$. For both diffuse and specular scattering, the collision integral is influenced by the potential function of interactions via the scattering angle, i.e.,

$$
\chi(g, b)=\pi-2 b \int_{r_{m}}^{\infty} \frac{d r}{r^{2} \sqrt{1-\frac{b^{2}}{r^{2}}-\frac{\Phi(r)}{m_{r} g^{2} / 2}}},
$$

where $r$ and $\Phi(r)$ are, respectively, the center-to-center separation and potential energy of interactions between the gas molecule and particle, and $r_{m}$ is the distance of the closest encounter.

For rigid-body collisions, $\Omega_{d}^{(1,1)^{*}} / \Omega_{s}^{(1,1) *}=1+\pi / 8$. For non-rigid-body collisions, this ratio is theoretically larger than unity [4]. As an example, Fig. 2 shows the variations of $\Omega_{s}^{(1,1)^{*}}$ and $\Omega_{d}^{(1,1)^{*}}$ as a function of the reduced temperature for a Lennard-Jones 12-6 potential. Here, the reduced temperature is given by $T^{*}=k T / \varepsilon$ [7], where $\varepsilon$ is the potentialenergy well depth.

While the Lennard-Jones 12-6 potential function is generally adequate for van der Waals interactions of molecular species, there is no evidence that the function is applicable 

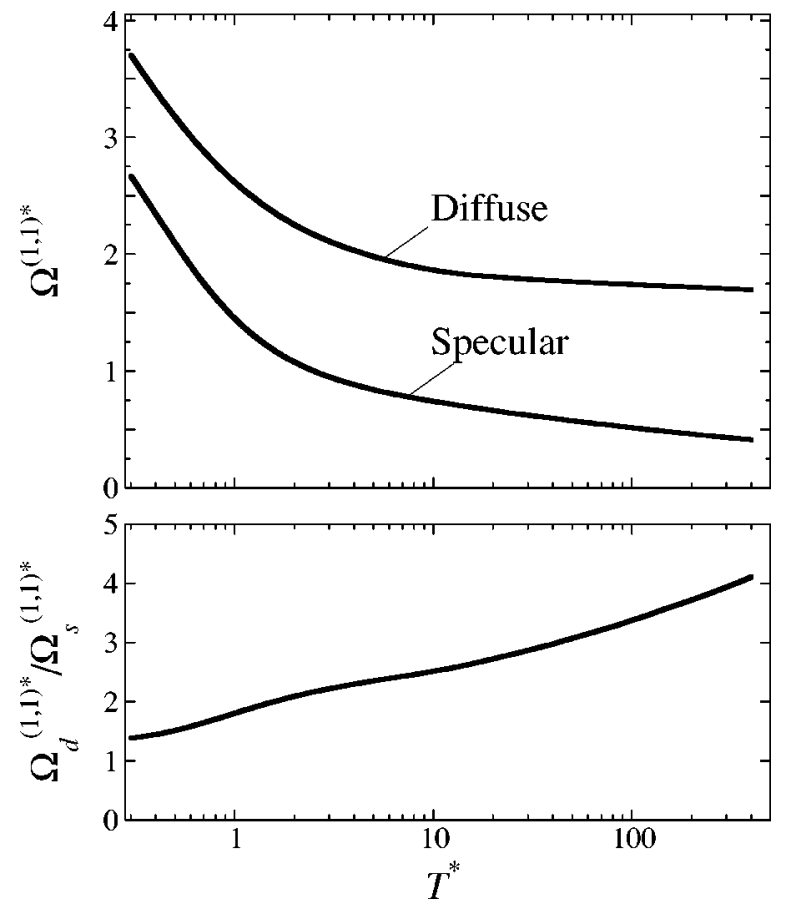

FIG. 2. Variations of the reduced collision integral $\Omega^{(1,1)^{*}}$ as a function of reduced temperature $T^{*}=k T / \varepsilon$ for the Lennard-Jones 12-6 potential function. The specular scattering result is taken from Hirschfelder et al. [7]; the diffuse scattering result was computed in the present work.

for the interactions of gas molecule with a particle. A potential function more relevant to molecule-particle interactions is perhaps the one recently proposed by Rudyak and Krasnolutski [10-12]. This potential function was constructed by summing the Lennard-Jones 12-6 potential energy of interaction between a fluid molecule with all atoms or molecules in the particle. Although there is some evidence that the dispersion forces are nonadditive [13], we nonetheless propose to use the Rudyak-Krasnolutski function because of lack of alternatives.

The Rudyak-Krasnolutski function considers a repulsive potential term $\Phi_{9}(r)$ in the short range and the attractive potential term $\Phi_{3}(r)$ in the long range of separations,

$$
\Phi(r)=\Phi_{9}(r)-\Phi_{3}(r)
$$

Here

$$
\begin{aligned}
\Phi_{i}(r)= & C_{i}\left\{\left[(r-R)^{-i}-(r+R)^{-i}\right]\right. \\
& \left.-a_{i}\left[(r-R)^{-i+1}-(r+R)^{-i+1}\right]\right\},
\end{aligned}
$$

where

$$
\begin{gathered}
a_{9}=9 r / 8, \\
a_{3}=3 r / 2, \\
C_{9}=4 \pi \varepsilon \sigma^{12} /(45 \mathrm{~V}), \\
C_{3}=2 \pi \varepsilon \sigma^{6} /(3 \mathrm{~V}),
\end{gathered}
$$

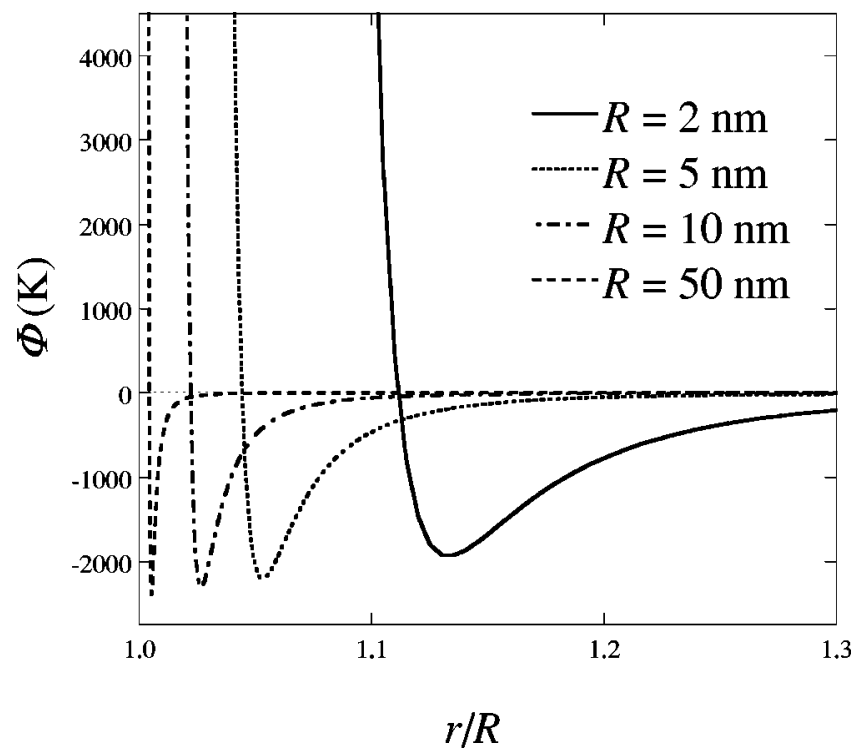

FIG. 3. The potential energy of interactions for a silver particle of radius $R$ and a nitrogen molecule as a function of the center-tocenter separation distance $r$. The potential energy is computed using a 9-3 potential function, Eq. (4) $\left(\sigma_{g}=3.652 \AA, \varepsilon_{g} / k\right.$ $=98.4 \mathrm{~K}, \sigma_{p}=2.54 \AA, \varepsilon_{p} / k=3995.4 \mathrm{~K}$, and $\left.\rho_{s}=10.5 \mathrm{~g} / \mathrm{cm}^{3}\right)$.

$V$ is the effective volume of the particle per molecule and may be related to the mass density of the particle $\rho_{s}$ by $V$ $=\bar{M} / \rho_{s}$, and $\bar{M}$ is the mean atomic mass of the particle material. The 9-3 potential function is related to molecular interactions via the parameters $\varepsilon$ and $\sigma$, i.e., the LennardJones 12-6 well-depth and collision diameter for the interactions of the fluid molecule and the constituent atom or molecule of the particle. Here the pair potential parameters may be estimated from the parameters of self-interactions through the well-accepted combination rules [14],

$$
\begin{gathered}
\sigma=\frac{\sigma_{g}+\sigma_{p}}{2}, \\
\varepsilon=\sqrt{\varepsilon_{g} \varepsilon_{p}},
\end{gathered}
$$

where the subscripts $g$ and $p$ denote the fluid molecule and constituent atom or molecule of the particle.

As an example, Fig. 3 shows the variation of the potential energy for the interaction of a nitrogen molecule with a silver particle, as a function of reduced separation $r / R$. The potential energy is computed using the Lennard-Jones 12-6 potential parameters of nitrogen molecule $\left(\sigma_{g}=3.652 \AA\right.$ and $\left.\varepsilon_{g} / k=98.4 \mathrm{~K}[15]\right)$, and silver atom $\left(\sigma_{p}=2.54 \AA\right.$ and $\left.\varepsilon_{p} / k=3995.4 \mathrm{~K}[16]\right)$. The mass density of the silver particle is $\rho_{s}=10.5 \mathrm{~g} / \mathrm{cm}^{3}$ [17] based on the assumption that it is identical to the bulk material. It is seen that the well depth is essentially independent of the particle radius. This is expected since $C_{3}$ and $C_{9}$ are essentially independent of the particle size if the effective volume $V$ is a constant. 
In defining the reduced collision integrals $\Omega_{s}^{(1,1)^{*}}$ and $\Omega_{d}^{(1,1)^{*}}$, we use $\pi R^{2}$ as the collision cross section [4] for the purpose of convenience. Obviously, the actual cross section must be larger than $\pi R^{2}$ [18] because of finite gas-molecule size. Following the approach of Fernández de la Mora et al. [18], an alternative choice can be made by using $\pi \sigma_{g-p}^{2}$, where $\sigma_{g-p}$ is the actual collision diameter. The collision diameter may be defined by the center-to-center separation corresponding to $\Phi\left(\sigma_{g-p}\right)=0$ or, simply, by a sum of the particle radius and one-half of the collision diameter of the gas molecule, i.e., $\sigma_{g-p}=R+\sigma_{g} / 2$. Then the $R^{2}$ term in Eq. (2) is replaced by $\sigma_{g-p}^{2}$. Theoretically this alternative approach is more rigorous, but practically the approach makes it very difficult to tabulate the collision integrals because they would have to be dependent on at least three independent variables, as compared to two independent variables if $\pi R^{2}$ is chosen as the cross section.

We note that practically the choice of cross section is inconsequential to the prediction of the drag force. The potential force of interactions affects the drag force primarily in the limit of small particle radius. It can be shown that the drag force is exact as long as the potential function is accurate and that the particle radius in Eq. (9) truthfully represents the particle size. In the large-particle-size limit, however, the choice of $\pi R^{2}$ theoretically introduces some inaccuracy, since the reduced collision integrals do not rigorously approach their asymptotic limits of rigid-sphere collisions. This, however, should not be a problem because (a) for large particles the potential force of interactions is unimportant and (b) $\pi \sigma_{g-p}^{2}$ naturally approaches $\pi R^{2}$ in the limit of large $R$.

Below we show that the reduced collision integrals given by Eq. (4) can be tabulated as a function of only two independent potential parameters. We introduce the dimensionless center-to-center separation as

$$
r^{\prime}=\frac{r}{R}
$$

and collision diameter as

$$
\sigma^{\prime}=\frac{\sigma}{R}
$$

We further introduce an effective well depth as

$$
\varepsilon^{\prime}=2 \pi \varepsilon \sigma^{3} / 3 V
$$

The potential energy is nondimensionalized as $\Phi^{\prime}\left(r^{\prime}\right)$ $=\Phi\left(r^{\prime}\right) / \varepsilon^{\prime}$ and

$$
\begin{aligned}
\Phi^{\prime}\left(r^{\prime}\right)= & \frac{2}{15} \sigma^{\prime 9}\left\{\frac{1}{\left(r^{\prime}-1\right)^{9}}-\frac{1}{\left(r^{\prime}+1\right)^{9}}-\frac{9}{8 r^{\prime}}\left[\frac{1}{\left(r^{\prime}-1\right)^{8}}\right.\right. \\
& \left.\left.-\frac{1}{\left(r^{\prime}+1\right)^{8}}\right]\right\}-\sigma^{\prime 3}\left\{\frac{1}{\left(r^{\prime}-1\right)^{3}}-\frac{1}{\left(r^{\prime}+1\right)^{3}}\right. \\
& \left.-\frac{3}{2 r^{\prime}}\left[\frac{1}{\left(r^{\prime}-1\right)^{2}}-\frac{1}{\left(r^{\prime}+1\right)^{2}}\right]\right\} .
\end{aligned}
$$

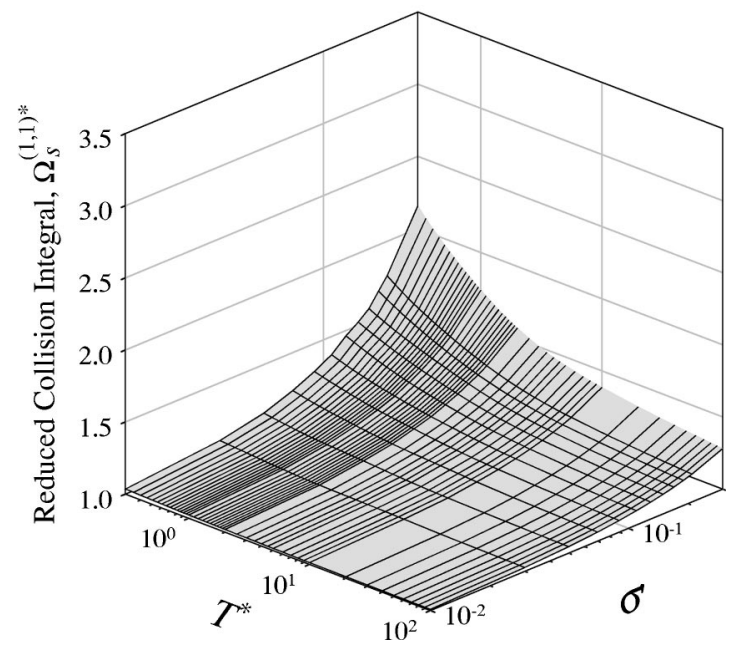

FIG. 4. Variation of the reduced collision integral for specular scattering as a function of modified reduced temperature $T^{*}$ $=k T / \varepsilon^{\prime}$ and the reduced collision diameter $\sigma^{\prime}$.

It is apparent that the collision integrals are functions of $\sigma^{\prime}$ and $\varepsilon^{\prime}$ only.

In Figs. 4 and 5 we plot the $\Omega_{s}^{(1,1)^{*}}$ and $\Omega_{d}^{(1,1)^{*}}$ values as functions of $\sigma^{\prime}$ and the modified reduced temperature $T^{*}$,

$$
T^{*}=\frac{k T}{\varepsilon^{\prime}}
$$

As expected, corrections to the rigid-body collision model are larger at lower temperatures and smaller particle radius, as evidenced by the larger $\Omega_{d(s)}^{(1,1) *}$ values at smaller $T^{*}$ and larger $\sigma^{\prime}$. Toward high temperatures and large particle radius, the collision integrals approach their respective rigidsphere limits of $\Omega_{d}^{(1,1)^{*}}=1+\pi / 8$ and $\Omega_{s}^{(1,1)^{*}}=1$. For practi-

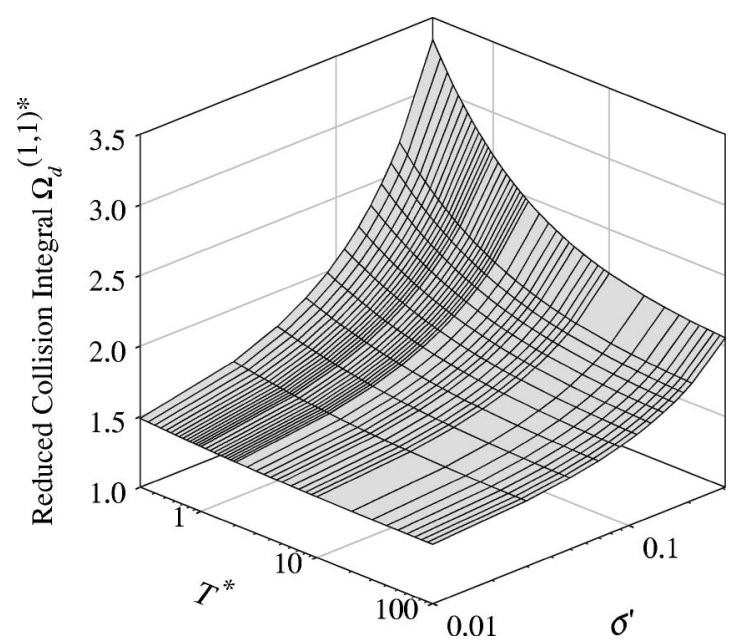

FIG. 5. Variation of the reduced collision integral for diffuse scattering as a function of modified reduced temperature $T^{*}$ $=k T / \varepsilon^{\prime}$ and the reduced collision diameter $\sigma^{\prime}$. 
TABLE I. Reduced collision integral $\Omega_{s}^{(1,1)^{*}}$.

\begin{tabular}{|c|c|c|c|c|c|c|c|c|c|c|c|c|c|c|c|}
\hline \multirow[b]{2}{*}{$T^{*}$} & \multicolumn{15}{|c|}{$\sigma^{\prime}$} \\
\hline & 0.01 & 0.03 & 0.05 & 0.07 & 0.09 & 0.11 & 0.13 & 0.15 & 0.17 & 0.19 & 0.2 & 0.3 & 0.4 & 0.5 & 0.6 \\
\hline 0.10 & 1.05 & 1.14 & 1.23 & 1.35 & 1.44 & 1.55 & 1.67 & 1.75 & 1.86 & 2.01 & 2.05 & 2.74 & 3.56 & 4.47 & 5.44 \\
\hline 0.15 & 1.04 & 1.12 & 1.20 & 1.30 & 1.37 & 1.47 & 1.58 & 1.65 & 1.74 & 1.87 & 1.91 & 2.47 & 3.16 & 3.91 & 4.68 \\
\hline 0.20 & 1.04 & 1.11 & 1.18 & 1.27 & 1.35 & 1.43 & 1.53 & 1.59 & 1.67 & 1.78 & 1.82 & 2.32 & 2.92 & 3.57 & 4.23 \\
\hline 0.25 & 1.04 & 1.10 & 1.17 & 1.25 & 1.32 & 1.40 & 1.49 & 1.55 & 1.62 & 1.72 & 1.76 & 2.21 & 2.75 & 3.34 & 3.92 \\
\hline 0.30 & 1.04 & 1.10 & 1.16 & 1.24 & 1.31 & 1.37 & 1.46 & 1.52 & 1.59 & 1.67 & 1.71 & 2.13 & 2.63 & 3.16 & 3.69 \\
\hline 0.35 & 1.03 & 1.10 & 1.16 & 1.22 & 1.29 & 1.35 & 1.44 & 1.49 & 1.56 & 1.64 & 1.67 & 2.07 & 2.53 & 3.02 & 3.51 \\
\hline 0.40 & 1.03 & 1.09 & 1.15 & 1.21 & 1.27 & 1.34 & 1.42 & 1.48 & 1.53 & 1.62 & 1.64 & 2.02 & 2.45 & 2.91 & 3.36 \\
\hline 0.45 & 1.03 & 1.08 & 1.14 & 1.21 & 1.27 & 1.32 & 1.40 & 1.46 & 1.52 & 1.59 & 1.62 & 1.98 & 2.38 & 2.81 & 3.23 \\
\hline 0.50 & 1.03 & 1.08 & 1.14 & 1.20 & 1.26 & 1.31 & 1.39 & 1.44 & 1.50 & 1.57 & 1.60 & 1.95 & 2.33 & 2.73 & 3.12 \\
\hline 0.55 & 1.03 & 1.08 & 1.13 & 1.19 & 1.25 & 1.30 & 1.38 & 1.43 & 1.49 & 1.55 & 1.58 & 1.92 & 2.28 & 2.65 & 3.03 \\
\hline 0.60 & 1.03 & 1.08 & 1.13 & 1.19 & 1.24 & 1.29 & 1.36 & 1.42 & 1.47 & 1.54 & 1.57 & 1.89 & 2.23 & 2.59 & 2.95 \\
\hline 0.65 & 1.03 & 1.08 & 1.13 & 1.18 & 1.24 & 1.29 & 1.35 & 1.41 & 1.46 & 1.52 & 1.55 & 1.86 & 2.19 & 2.53 & 2.87 \\
\hline 0.70 & 1.03 & 1.07 & 1.12 & 1.18 & 1.23 & 1.28 & 1.35 & 1.40 & 1.45 & 1.51 & 1.54 & 1.84 & 2.16 & 2.48 & 2.81 \\
\hline 0.75 & 1.02 & 1.07 & 1.12 & 1.18 & 1.23 & 1.28 & 1.34 & 1.39 & 1.44 & 1.50 & 1.53 & 1.82 & 2.12 & 2.43 & 2.75 \\
\hline 0.80 & 1.02 & 1.07 & 1.12 & 1.17 & 1.22 & 1.27 & 1.33 & 1.38 & 1.43 & 1.49 & 1.52 & 1.80 & 2.09 & 2.39 & 2.70 \\
\hline 0.85 & 1.02 & 1.07 & 1.12 & 1.17 & 1.22 & 1.27 & 1.32 & 1.38 & 1.43 & 1.48 & 1.51 & 1.78 & 2.06 & 2.36 & 2.65 \\
\hline 0.90 & 1.02 & 1.07 & 1.12 & 1.17 & 1.21 & 1.26 & 1.32 & 1.37 & 1.42 & 1.47 & 1.50 & 1.77 & 2.04 & 2.32 & 2.61 \\
\hline 0.95 & 1.02 & 1.07 & 1.11 & 1.16 & 1.21 & 1.25 & 1.31 & 1.36 & 1.41 & 1.46 & 1.49 & 1.75 & 2.02 & 2.29 & 2.57 \\
\hline 1.00 & 1.02 & 1.07 & 1.11 & 1.16 & 1.21 & 1.25 & 1.31 & 1.36 & 1.41 & 1.45 & 1.48 & 1.74 & 1.99 & 2.26 & 2.53 \\
\hline 1.10 & 1.02 & 1.06 & 1.11 & 1.16 & 1.20 & 1.25 & 1.30 & 1.35 & 1.40 & 1.44 & 1.47 & 1.71 & 1.96 & 2.21 & 2.47 \\
\hline 1.20 & 1.02 & 1.06 & 1.11 & 1.15 & 1.20 & 1.24 & 1.29 & 1.34 & 1.38 & 1.43 & 1.46 & 1.69 & 1.92 & 2.16 & 2.41 \\
\hline 1.30 & 1.02 & 1.06 & 1.11 & 1.15 & 1.19 & 1.23 & 1.28 & 1.33 & 1.38 & 1.42 & 1.44 & 1.67 & 1.89 & 2.13 & 2.36 \\
\hline 1.40 & 1.02 & 1.06 & 1.10 & 1.14 & 1.19 & 1.23 & 1.28 & 1.32 & 1.37 & 1.41 & 1.43 & 1.65 & 1.87 & 2.09 & 2.32 \\
\hline 1.50 & 1.02 & 1.06 & 1.10 & 1.14 & 1.19 & 1.22 & 1.27 & 1.31 & 1.36 & 1.40 & 1.42 & 1.63 & 1.84 & 2.06 & 2.29 \\
\hline 1.60 & 1.02 & 1.06 & 1.10 & 1.14 & 1.18 & 1.22 & 1.27 & 1.31 & 1.35 & 1.39 & 1.41 & 1.62 & 1.82 & 2.04 & 2.26 \\
\hline 1.70 & 1.02 & 1.06 & 1.10 & 1.14 & 1.18 & 1.22 & 1.26 & 1.30 & 1.34 & 1.38 & 1.41 & 1.60 & 1.81 & 2.01 & 2.23 \\
\hline 1.80 & 1.02 & 1.06 & 1.10 & 1.14 & 1.18 & 1.21 & 1.26 & 1.30 & 1.34 & 1.37 & 1.40 & 1.59 & 1.79 & 1.99 & 2.20 \\
\hline 1.90 & 1.02 & 1.05 & 1.10 & 1.13 & 1.17 & 1.21 & 1.25 & 1.29 & 1.33 & 1.37 & 1.39 & 1.58 & 1.77 & 1.97 & 2.18 \\
\hline 2.00 & 1.02 & 1.05 & 1.10 & 1.13 & 1.17 & 1.21 & 1.25 & 1.29 & 1.33 & 1.36 & 1.38 & 1.57 & 1.76 & 1.96 & 2.16 \\
\hline 3.00 & 1.02 & 1.05 & 1.09 & 1.12 & 1.15 & 1.19 & 1.22 & 1.25 & 1.29 & 1.32 & 1.34 & 1.50 & 1.67 & 1.84 & 2.03 \\
\hline 4.00 & 1.02 & 1.05 & 1.08 & 1.11 & 1.14 & 1.17 & 1.20 & 1.23 & 1.26 & 1.29 & 1.31 & 1.46 & 1.62 & 1.79 & 1.96 \\
\hline 5.00 & 1.01 & 1.05 & 1.07 & 1.11 & 1.13 & 1.17 & 1.19 & 1.22 & 1.25 & 1.28 & 1.29 & 1.44 & 1.59 & 1.75 & 1.91 \\
\hline 6.00 & 1.01 & 1.05 & 1.07 & 1.10 & 1.13 & 1.16 & 1.18 & 1.21 & 1.23 & 1.27 & 1.28 & 1.42 & 1.57 & 1.72 & 1.88 \\
\hline 7.00 & 1.01 & 1.04 & 1.07 & 1.10 & 1.12 & 1.15 & 1.18 & 1.20 & 1.23 & 1.26 & 1.27 & 1.41 & 1.55 & 1.70 & 1.86 \\
\hline 8.00 & 1.01 & 1.04 & 1.07 & 1.10 & 1.12 & 1.15 & 1.17 & 1.19 & 1.22 & 1.25 & 1.26 & 1.40 & 1.54 & 1.68 & 1.84 \\
\hline 9.00 & 1.01 & 1.04 & 1.06 & 1.09 & 1.11 & 1.14 & 1.17 & 1.19 & 1.22 & 1.24 & 1.26 & 1.39 & 1.53 & 1.67 & 1.82 \\
\hline 10.00 & 1.01 & 1.04 & 1.06 & 1.09 & 1.11 & 1.14 & 1.16 & 1.19 & 1.21 & 1.24 & 1.25 & 1.38 & 1.52 & 1.66 & 1.81 \\
\hline 20.00 & 1.01 & 1.03 & 1.06 & 1.08 & 1.10 & 1.12 & 1.15 & 1.17 & 1.19 & 1.21 & 1.23 & 1.34 & 1.47 & 1.60 & 1.73 \\
\hline 30.00 & 1.01 & 1.03 & 1.05 & 1.07 & 1.09 & 1.12 & 1.14 & 1.16 & 1.18 & 1.20 & 1.21 & 1.33 & 1.44 & 1.56 & 1.69 \\
\hline 40.00 & 1.01 & 1.03 & 1.05 & 1.07 & 1.09 & 1.11 & 1.13 & 1.15 & 1.17 & 1.19 & 1.21 & 1.31 & 1.43 & 1.54 & 1.67 \\
\hline 50.00 & 1.01 & 1.03 & 1.05 & 1.07 & 1.09 & 1.11 & 1.13 & 1.15 & 1.17 & 1.19 & 1.20 & 1.31 & 1.41 & 1.53 & 1.65 \\
\hline 60.00 & 1.01 & 1.03 & 1.05 & 1.07 & 1.09 & 1.11 & 1.13 & 1.14 & 1.16 & 1.18 & 1.20 & 1.30 & 1.41 & 1.52 & 1.63 \\
\hline 70.00 & 1.01 & 1.03 & 1.05 & 1.06 & 1.08 & 1.10 & 1.12 & 1.14 & 1.16 & 1.18 & 1.19 & 1.29 & 1.40 & 1.51 & 1.62 \\
\hline 80.00 & 1.01 & 1.03 & 1.05 & 1.06 & 1.08 & 1.10 & 1.12 & 1.14 & 1.16 & 1.18 & 1.19 & 1.29 & 1.39 & 1.50 & 1.61 \\
\hline 90.00 & 1.01 & 1.03 & 1.05 & 1.06 & 1.08 & 1.10 & 1.12 & 1.14 & 1.16 & 1.18 & 1.19 & 1.28 & 1.39 & 1.49 & 1.60 \\
\hline 100.00 & 1.01 & 1.03 & 1.04 & 1.06 & 1.08 & 1.09 & 1.12 & 1.14 & 1.15 & 1.17 & 1.18 & 1.27 & 1.38 & 1.48 & 1.59 \\
\hline
\end{tabular}

cal uses, the $\Omega_{s}^{(1,1)^{*}}$ and $\Omega_{d}^{(1,1)^{*}}$ values are tabulated in Tables I and II, respectively, as functions of $\sigma^{\prime}$ and $T^{*}$. The ranges of $T^{*}(<100)$ and $\sigma^{\prime}(<0.6)$ values were chosen to be sufficiently wide for all practical purposes. The maximum $\sigma^{\prime}$ value ensures that the collision integral is applicable to particles with radius as small as $0.5 \mathrm{~nm}$.

The reduced collision integral values may be parametrized by the following two equations: 
TABLE II. Reduced collision integral $\Omega_{d}^{(1,1)^{*}}$.

\begin{tabular}{|c|c|c|c|c|c|c|c|c|c|c|c|c|c|c|c|}
\hline \multirow[b]{2}{*}{$T^{*}$} & \multicolumn{15}{|c|}{$\sigma^{\prime}$} \\
\hline & 0.01 & 0.03 & 0.05 & 0.07 & 0.09 & 0.11 & 0.13 & 0.15 & 0.17 & 0.19 & 0.2 & 0.3 & 0.4 & 0.5 & 0.6 \\
\hline 0.10 & 1.52 & 1.70 & 1.87 & 2.04 & 2.22 & 2.38 & 2.56 & 2.73 & 2.92 & 3.10 & 3.20 & 4.20 & 5.32 & 6.57 & 7.91 \\
\hline 0.15 & 1.51 & 1.67 & 1.82 & 1.97 & 2.12 & 2.27 & 2.41 & 2.57 & 2.72 & 2.88 & 2.96 & 3.81 & 4.75 & 5.79 & 6.90 \\
\hline 0.20 & 1.49 & 1.65 & 1.78 & 1.92 & 2.06 & 2.19 & 2.33 & 2.46 & 2.61 & 2.75 & 2.82 & 3.58 & 4.41 & 5.31 & 6.29 \\
\hline 0.25 & 1.49 & 1.63 & 1.76 & 1.89 & 2.02 & 2.14 & 2.26 & 2.39 & 2.52 & 2.65 & 2.72 & 3.41 & 4.17 & 4.99 & 5.86 \\
\hline 0.30 & 1.48 & 1.62 & 1.74 & 1.86 & 1.98 & 2.10 & 2.22 & 2.34 & 2.46 & 2.58 & 2.64 & 3.29 & 3.99 & 4.74 & 5.55 \\
\hline 0.35 & 1.48 & 1.61 & 1.73 & 1.84 & 1.96 & 2.07 & 2.18 & 2.29 & 2.41 & 2.52 & 2.58 & 3.20 & 3.85 & 4.55 & 5.29 \\
\hline 0.40 & 1.48 & 1.60 & 1.72 & 1.83 & 1.94 & 2.04 & 2.15 & 2.26 & 2.37 & 2.48 & 2.53 & 3.12 & 3.74 & 4.39 & 5.09 \\
\hline 0.45 & 1.47 & 1.60 & 1.71 & 1.81 & 1.92 & 2.02 & 2.12 & 2.23 & 2.33 & 2.44 & 2.49 & 3.05 & 3.64 & 4.26 & 4.91 \\
\hline 0.50 & 1.47 & 1.59 & 1.70 & 1.80 & 1.90 & 2.00 & 2.10 & 2.20 & 2.30 & 2.40 & 2.46 & 2.99 & 3.56 & 4.14 & 4.76 \\
\hline 0.55 & 1.47 & 1.59 & 1.69 & 1.79 & 1.89 & 1.98 & 2.08 & 2.18 & 2.28 & 2.38 & 2.43 & 2.94 & 3.48 & 4.04 & 4.63 \\
\hline 0.60 & 1.47 & 1.58 & 1.68 & 1.78 & 1.88 & 1.97 & 2.06 & 2.16 & 2.26 & 2.35 & 2.40 & 2.90 & 3.42 & 3.95 & 4.52 \\
\hline 0.65 & 1.47 & 1.58 & 1.68 & 1.77 & 1.87 & 1.96 & 2.05 & 2.14 & 2.24 & 2.33 & 2.37 & 2.86 & 3.36 & 3.88 & 4.42 \\
\hline 0.70 & 1.47 & 1.57 & 1.67 & 1.76 & 1.86 & 1.94 & 2.03 & 2.12 & 2.22 & 2.31 & 2.35 & 2.82 & 3.31 & 3.81 & 4.33 \\
\hline 0.75 & 1.47 & 1.57 & 1.67 & 1.76 & 1.85 & 1.93 & 2.02 & 2.11 & 2.20 & 2.29 & 2.33 & 2.79 & 3.26 & 3.74 & 4.25 \\
\hline 0.80 & 1.47 & 1.57 & 1.66 & 1.75 & 1.84 & 1.92 & 2.01 & 2.10 & 2.19 & 2.27 & 2.31 & 2.76 & 3.22 & 3.68 & 4.18 \\
\hline 0.85 & 1.47 & 1.56 & 1.66 & 1.74 & 1.83 & 1.91 & 2.00 & 2.09 & 2.17 & 2.25 & 2.30 & 2.73 & 3.18 & 3.63 & 4.11 \\
\hline 0.90 & 1.47 & 1.56 & 1.65 & 1.74 & 1.82 & 1.91 & 1.99 & 2.07 & 2.16 & 2.24 & 2.28 & 2.71 & 3.14 & 3.59 & 4.05 \\
\hline 0.95 & 1.47 & 1.56 & 1.65 & 1.73 & 1.82 & 1.90 & 1.98 & 2.06 & 2.15 & 2.23 & 2.27 & 2.68 & 3.11 & 3.54 & 4.00 \\
\hline 1.00 & 1.47 & 1.56 & 1.64 & 1.73 & 1.81 & 1.89 & 1.97 & 2.05 & 2.14 & 2.21 & 2.25 & 2.66 & 3.08 & 3.50 & 3.95 \\
\hline 1.10 & 1.47 & 1.55 & 1.64 & 1.72 & 1.80 & 1.88 & 1.96 & 2.04 & 2.12 & 2.19 & 2.23 & 2.62 & 3.02 & 3.43 & 3.86 \\
\hline 1.20 & 1.46 & 1.55 & 1.63 & 1.71 & 1.79 & 1.87 & 1.94 & 2.02 & 2.10 & 2.17 & 2.21 & 2.59 & 2.98 & 3.37 & 3.79 \\
\hline 1.30 & 1.46 & 1.54 & 1.63 & 1.70 & 1.78 & 1.86 & 1.93 & 2.01 & 2.08 & 2.15 & 2.19 & 2.56 & 2.93 & 3.32 & 3.72 \\
\hline 1.40 & 1.46 & 1.54 & 1.62 & 1.70 & 1.78 & 1.85 & 1.92 & 1.99 & 2.07 & 2.14 & 2.17 & 2.53 & 2.90 & 3.27 & 3.67 \\
\hline 1.50 & 1.46 & 1.54 & 1.62 & 1.69 & 1.77 & 1.84 & 1.91 & 1.98 & 2.05 & 2.12 & 2.16 & 2.51 & 2.86 & 3.23 & 3.62 \\
\hline 1.60 & 1.46 & 1.54 & 1.61 & 1.69 & 1.76 & 1.83 & 1.90 & 1.97 & 2.04 & 2.11 & 2.14 & 2.48 & 2.83 & 3.20 & 3.58 \\
\hline 1.70 & 1.46 & 1.54 & 1.61 & 1.68 & 1.76 & 1.82 & 1.89 & 1.96 & 2.03 & 2.09 & 2.13 & 2.46 & 2.81 & 3.16 & 3.54 \\
\hline 1.80 & 1.46 & 1.53 & 1.61 & 1.68 & 1.75 & 1.82 & 1.88 & 1.95 & 2.02 & 2.08 & 2.12 & 2.45 & 2.78 & 3.13 & 3.50 \\
\hline 1.90 & 1.46 & 1.53 & 1.61 & 1.68 & 1.74 & 1.81 & 1.88 & 1.94 & 2.01 & 2.07 & 2.10 & 2.43 & 2.76 & 3.11 & 3.47 \\
\hline 2.00 & 1.45 & 1.53 & 1.60 & 1.67 & 1.74 & 1.80 & 1.87 & 1.94 & 2.00 & 2.06 & 2.09 & 2.41 & 2.74 & 3.09 & 3.44 \\
\hline 3.00 & 1.44 & 1.52 & 1.58 & 1.64 & 1.70 & 1.76 & 1.82 & 1.88 & 1.94 & 1.99 & 2.02 & 2.31 & 2.61 & 2.93 & 3.27 \\
\hline 4.00 & 1.43 & 1.51 & 1.57 & 1.62 & 1.68 & 1.74 & 1.79 & 1.84 & 1.90 & 1.95 & 1.98 & 2.26 & 2.55 & 2.85 & 3.18 \\
\hline 5.00 & 1.43 & 1.50 & 1.56 & 1.61 & 1.67 & 1.72 & 1.77 & 1.82 & 1.87 & 1.93 & 1.95 & 2.22 & 2.50 & 2.80 & 3.12 \\
\hline 6.00 & 1.43 & 1.49 & 1.55 & 1.60 & 1.66 & 1.71 & 1.76 & 1.81 & 1.86 & 1.91 & 1.93 & 2.20 & 2.48 & 2.77 & 3.08 \\
\hline 7.00 & 1.42 & 1.49 & 1.54 & 1.60 & 1.65 & 1.70 & 1.74 & 1.79 & 1.85 & 1.90 & 1.92 & 2.18 & 2.45 & 2.75 & 3.05 \\
\hline 8.00 & 1.42 & 1.48 & 1.54 & 1.59 & 1.64 & 1.69 & 1.73 & 1.78 & 1.84 & 1.89 & 1.91 & 2.17 & 2.44 & 2.73 & 3.03 \\
\hline 9.00 & 1.42 & 1.48 & 1.54 & 1.59 & 1.64 & 1.68 & 1.73 & 1.78 & 1.83 & 1.88 & 1.90 & 2.16 & 2.43 & 2.71 & 3.02 \\
\hline 10.00 & 1.42 & 1.47 & 1.52 & 1.57 & 1.62 & 1.67 & 1.72 & 1.77 & 1.82 & 1.87 & 1.89 & 2.15 & 2.42 & 2.70 & 3.00 \\
\hline 20.00 & 1.42 & 1.46 & 1.51 & 1.55 & 1.60 & 1.65 & 1.69 & 1.74 & 1.79 & 1.84 & 1.87 & 2.11 & 2.37 & 2.65 & 2.94 \\
\hline 30.00 & 1.41 & 1.46 & 1.50 & 1.55 & 1.59 & 1.64 & 1.68 & 1.73 & 1.78 & 1.82 & 1.85 & 2.09 & 2.34 & 2.61 & 2.90 \\
\hline 40.00 & 1.41 & 1.46 & 1.50 & 1.54 & 1.59 & 1.63 & 1.68 & 1.72 & 1.77 & 1.82 & 1.84 & 2.08 & 2.33 & 2.59 & 2.88 \\
\hline 50.00 & 1.41 & 1.46 & 1.50 & 1.54 & 1.59 & 1.63 & 1.67 & 1.72 & 1.77 & 1.81 & 1.83 & 2.06 & 2.31 & 2.58 & 2.86 \\
\hline 60.00 & 1.41 & 1.46 & 1.50 & 1.54 & 1.58 & 1.63 & 1.67 & 1.72 & 1.76 & 1.81 & 1.83 & 2.06 & 2.31 & 2.57 & 2.85 \\
\hline 70.00 & 1.41 & 1.45 & 1.50 & 1.54 & 1.58 & 1.63 & 1.67 & 1.71 & 1.76 & 1.81 & 1.82 & 2.05 & 2.30 & 2.56 & 2.84 \\
\hline 80.00 & 1.41 & 1.45 & 1.50 & 1.54 & 1.58 & 1.62 & 1.67 & 1.71 & 1.76 & 1.80 & 1.81 & 2.05 & 2.29 & 2.56 & 2.84 \\
\hline 90.00 & 1.41 & 1.45 & 1.50 & 1.54 & 1.58 & 1.62 & 1.67 & 1.71 & 1.76 & 1.80 & 1.81 & 2.04 & 2.29 & 2.55 & 2.83 \\
\hline 100.00 & 1.41 & 1.45 & 1.50 & 1.54 & 1.58 & 1.62 & 1.67 & 1.71 & 1.76 & 1.80 & 1.81 & 2.04 & 2.29 & 2.55 & 2.83 \\
\hline
\end{tabular}

$$
\begin{aligned}
\Omega_{d}^{(1,1)^{*}=} & 1+\frac{\pi}{8}+\left[1.072+\frac{2.078}{T^{* 1 / 4}}+\frac{1.261}{T^{* 1 / 2}}\right] \sigma^{\prime} \\
& +\left[3.285-\frac{8.872}{T^{* 1 / 4}}+\frac{5.225}{T^{* 1 / 2}}\right] \sigma^{\prime 2}
\end{aligned}
$$

and

$$
\begin{aligned}
\Omega_{s}^{(1,1)^{*}=} & 1+\left[0.316+\frac{1.47}{T^{* 1 / 4}}+\frac{0.476}{T^{* 1 / 2}}\right] \sigma^{\prime} \\
& +\left[1.53-\frac{5.013}{T^{* 1 / 4}}+\frac{4.025}{T^{* 1 / 2}}\right] \sigma^{\prime 2} .
\end{aligned}
$$

Both equations satisfy the asymptotic limits of rigid-sphere 
collisions at $T^{*} \rightarrow \infty$ and/or $\sigma^{\prime} \rightarrow 0$. The maximum fitting errors are $4.4 \%$ and $3.3 \%$ for Eqs. (17) and (18), respectively. Both occur for large $\sigma^{\prime}$ and small $T^{*}$, i.e., the high curvature areas shown in Figs. 4 and 5. In other areas, the fitting error is well within $2 \%$.

\section{MOMENTUM-ACCOMMODATION FUNCTION}

As discussed earlier, the momentum-accommodation function $\varphi$ essentially describes, with an increase in the particle size, the transition of the dominant outcome of collision between a gas molecule and a particle from specular scattering to diffuse scattering. Based on experimental and theoretical evidence, $\varphi$ should assume the asymptotic values of $\sim 1$ and 0 in the limits of large and small particle sizes, respectively [4]. The transition is expected to occur at a particle diameter of a few nanometers [19]. Beyond the aforementioned evidence, little is known about the shape and form of the momentum-accommodation function. Therefore we are forced to treat this function empirically and propose that this function be modeled by the following equation:

$$
\varphi=\frac{1+0.9 \operatorname{Kn}\left\{1-1 /\left[1+(R / \beta)^{\alpha}\right]\right\}}{1+\mathrm{Kn}} .
$$

Clearly Eq. (19) reduces to unity for $\mathrm{Kn} \ll 1$. This limiting condition is found to be important to drag-force prediction in the transition regime, which will be discussed in Sec. V. For $\mathrm{Kn} \gg 1$, Eq. (19) is reduced to $\varphi=0.9\left\{1-1 /\left[1+(R / \beta)^{\alpha}\right]\right\}$. Here the constant 0.9 ensures that the scattering approaches the diffuse-to-specular ratio of 90 to 10 for large $R$ values, in agreement with Millikan's observation [3]. The constant $\beta$ is related to the particle size at the transition from specular to diffuse scattering. The curvature or sharpness of the transition is determined by constant $\alpha$. These empirical constants are subsequently determined by fitting them against available experimental data, as will be discussed below.

In recent years there have been several studies [12,20,21] that compared mobility sizes measured by the differential mobility analyzer (DMA) with sizes measured by transmission electronic microscopy (TEM). These measurements were made for nearly spherical silver particles in nitrogen [20,21] and copper oxide particles again in nitrogen [12]. The particles are as small as $1.7 \mathrm{~nm}$ in radius. These data are reproduced in Table III. On average, the mobility size was found to be larger than the TEM size by about $20 \%$. This is expected since the DMA measurements used the StokesCunningham formula (1), which is inherently consistent with the diffuse-scattering limit and does not consider the influence of potential force of interactions between the fluid molecule and particle.

The experimental data just discussed are used here to obtain the constants in Eq. (19). Specifically, the "experimental" drag coefficients $k_{d}=-\mathbf{F} / \mathbf{V}$ are derived from the mobility radius $R_{\mathrm{DMA}}$ for each experiment given in Table III, using the Stokes-Cunningham formula (1). The viscosity of nitrogen was appropriately chosen for the temperature of $294 \mathrm{~K}$ and under 1 atm pressure and equal to $1.78 \times 10^{-4} \mathrm{cp}$
TABLE III. Particle sizes (nm) measured by TEM, DMA, and predicted by the current theory.

\begin{tabular}{|c|c|c|c|c|}
\hline$R_{\mathrm{TEM}}$ & $R_{\mathrm{DMA}}$ & Error $(\%)$ & $R_{\text {Theory }}$ & Error $(\%)$ \\
\hline \multicolumn{5}{|l|}{$\mathrm{Ag}[20]$} \\
\hline 11.15 & 13.75 & 23.3 & 12.06 & 8.2 \\
\hline 9.45 & 11.05 & 16.9 & 9.60 & 1.6 \\
\hline 7.20 & 7.70 & 6.9 & 6.61 & -8.2 \\
\hline 6.85 & 8.10 & 18.2 & 6.91 & 0.8 \\
\hline 6.65 & 9.75 & 46.6 & 8.25 & 24.1 \\
\hline 6.45 & 8.10 & 25.6 & 6.87 & 6.5 \\
\hline 5.85 & 6.60 & 12.8 & 5.57 & -4.8 \\
\hline 5.20 & 6.60 & 26.9 & 5.49 & 5.6 \\
\hline 4.98 & 5.38 & 8.0 & 4.47 & -10.3 \\
\hline 4.97 & 6.85 & 37.8 & 5.65 & 13.7 \\
\hline 4.94 & 5.90 & 19.6 & 4.88 & -1.1 \\
\hline 4.27 & 5.38 & 26.0 & 4.38 & 2.5 \\
\hline 3.97 & 4.20 & 5.8 & 3.39 & -14.6 \\
\hline 3.94 & 3.41 & -13.3 & 2.76 & -29.8 \\
\hline 3.42 & 4.20 & 22.8 & 3.33 & -2.5 \\
\hline 3.30 & 4.81 & 45.8 & 3.79 & 14.8 \\
\hline 3.08 & 4.20 & 36.6 & 3.31 & 7.7 \\
\hline 2.71 & 3.41 & 26.1 & 2.82 & 4.1 \\
\hline 2.62 & 3.41 & 30.0 & 2.86 & 9.0 \\
\hline 1.93 & 2.43 & 26.0 & 2.09 & 8.5 \\
\hline \multirow[t]{2}{*}{1.66} & 2.40 & 45.0 & 1.99 & 20.4 \\
\hline & Mean error & 23.5 & & 9.5 \\
\hline \multicolumn{5}{|l|}{$\mathrm{Ag}[21]$} \\
\hline 10.74 & 12.27 & 14.3 & 11.05 & 3.0 \\
\hline 8.04 & 9.99 & 24.3 & 8.76 & 9.1 \\
\hline 7.78 & 8.14 & 4.6 & 7.13 & -8.3 \\
\hline 6.14 & 6.65 & 8.2 & 5.71 & -6.9 \\
\hline 3.76 & 5.38 & 42.8 & 4.33 & 15.2 \\
\hline 3.66 & 4.40 & 20.1 & 3.53 & -3.5 \\
\hline \multirow[t]{2}{*}{2.97} & 3.61 & 21.8 & 2.90 & -2.2 \\
\hline & Mean error & 19.4 & & 6.9 \\
\hline \multicolumn{5}{|l|}{$\mathrm{Cu}_{2} \mathrm{O}$ [12] } \\
\hline 4.47 & 5.63 & 26.0 & 4.52 & 1.2 \\
\hline 3.77 & 4.59 & 21.8 & 3.60 & -4.5 \\
\hline 3.16 & 3.74 & 18.4 & 2.89 & -8.5 \\
\hline 2.92 & 3.38 & 15.6 & 2.63 & -9.8 \\
\hline 2.68 & 3.05 & 14.0 & 2.49 & -7.1 \\
\hline 2.40 & 2.76 & 15.0 & 2.34 & -2.1 \\
\hline 2.26 & 2.49 & 10.4 & 2.12 & -5.9 \\
\hline 1.94 & 2.25 & 16.3 & 1.87 & -3.2 \\
\hline 1.67 & 2.03 & 21.9 & 1.63 & -2.2 \\
\hline 1.47 & 1.84 & 24.8 & 1.41 & -3.8 \\
\hline \multirow[t]{2}{*}{1.28} & 1.66 & 30.2 & 1.22 & -4.5 \\
\hline & Mean error & 19.5 & & 4.8 \\
\hline
\end{tabular}

$[17,22]$. The $A$ and $B$ values are the same as those used in the original work, equal to 1.257 and 0.4 , respectively $[12,21]$.

The values of $\alpha$ and $\beta$ are obtained by a best fit to the 
TABLE IV. Lennard-Jones 12-6 parameters for the potential energy of self-interactions and the mass density of particle materials used in the analysis.

\begin{tabular}{cccc}
\hline \hline Atom (molecule) & $\sigma_{g}(\AA)$ & $\varepsilon_{g}(\mathrm{~K})$ & Reference/comments \\
\hline $\mathrm{N}_{2}$ & 3.652 & 98.4 & {$[15]$} \\
$\mathrm{Air}$ & 3.652 & 98.4 & Same as $\mathrm{N}_{2}$ \\
& $\sigma_{p}(\AA)$ & $\varepsilon_{p}(\mathrm{~K})$ & \\
\hline $\mathrm{Ag}$ & 2.540 & 3995.4 & {$[16]$} \\
$\mathrm{Cu} 2 \mathrm{O}$ & 4.124 & 2909.1 & {$[12]$} \\
$\mathrm{C}(\mathrm{protein})$ & 3.500 & 33.3 & {$[23]$} \\
& $\rho_{s}\left(\mathrm{~g} / \mathrm{cm}^{3}\right)$ & & \\
& 10.5 & & {$[17]$} \\
$\mathrm{Ag}$ & 6.1 & & {$[12]$} \\
$\mathrm{Cu}_{2} \mathrm{O}$ & 1.28 & & {$[18]$} \\
$\mathrm{Protein}$ & & & \\
\hline \hline
\end{tabular}

three sets of "experimental" drag coefficients by combining Eqs. (2), (3), and (19). The collision integrals $\Omega_{s}^{(1,1)^{*}}$ and $\Omega_{d}^{(1,1)^{*}}$ are determined using the Lennard-Jones potential function parameters and the mass density values shown in Table IV. The combination rules [Eqs. (10) and (11)] are used to determine $\sigma_{g-p}$. The best fit gives

$$
\varphi=\frac{1+0.9 \operatorname{Kn}\left\{1-1 /\left[1+(R / 2.5)^{15}\right]\right\}}{1+\mathrm{Kn}}
$$

where $R$ is in units of nm. Table III and Fig. 6 show the comparison of the radius predicted by the current theory and the TEM radius. It is seen that the theory predicts well the

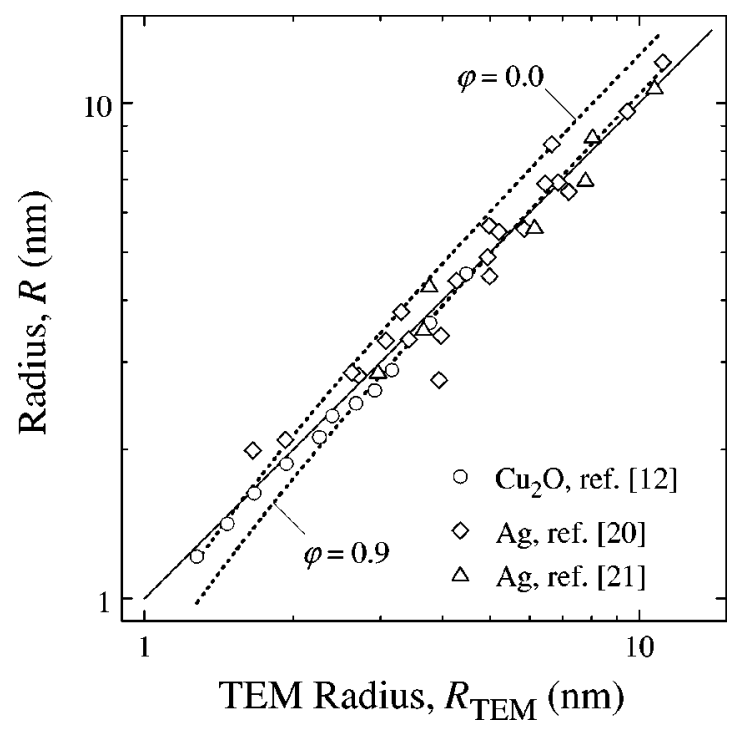

FIG. 6. Comparison of particle radius determined by the current theory and the TEM radius. The dashed lines illustrate the predictions using the limiting cases of $\varphi=0$ (specular scattering) and $\varphi=0.9$ (predominantly diffuse scattering).

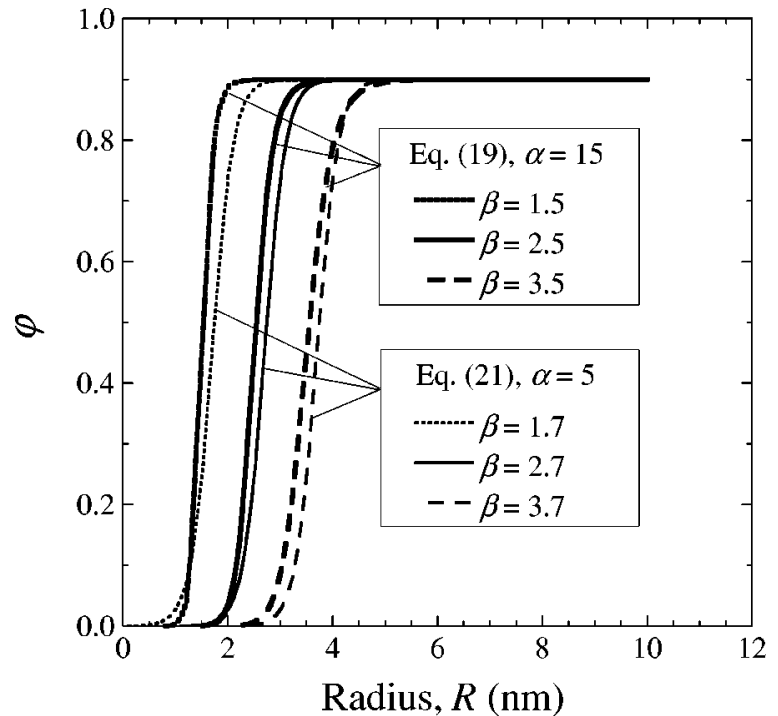

FIG. 7. Samples of the momentum-accommodation function used in the current analysis.

TEM radius over the entire range of radius. The mean error in the predicted radius is $7.7 \%$, substantially better than the Stokes-Cunningham formula.

We examined the sensitivity of the drag-force prediction with respect to the form of the momentum-accommodation function and its parameters. Figure 7 shows two sets of sample momentum-accommodation functions used in the analysis. The first set is given by Eq. (19) with the constants $\alpha$ and $\beta$ given in Fig. 7. In the second set, we used a slightly different function, given by

$$
\varphi=\frac{1+0.9 \mathrm{Kn}\left[1+e^{-\alpha(R-\beta)}\right]}{1+\mathrm{Kn}},
$$

where $\alpha$ and $\beta$ are again constants and listed Fig. 7. Table V presents a comparison of the percentage errors of particle radius predicted by these functions, along with several other

TABLE V. Sensitivity of the theory to the parameters in momentum-accommodation function.

\begin{tabular}{lcccc}
\hline \hline & Case & $\alpha$ & $\beta$ (nm) & Mean error $(\%)$ \\
\hline Eq. (19) & & & & \\
& 1 & 15 & 1.5 & 9.34 \\
& 2 & 15 & 2.5 & 7.84 \\
& 3 & 15 & 3.5 & 9.02 \\
& 4 & 10 & 2.5 & 7.85 \\
& 5 & 20 & 2.5 & 7.84 \\
Eq. (21) & 6 & 50 & 2.5 & 7.77 \\
& 1 & & & \\
& 2 & 5 & 1.7 & 9.10 \\
& 3 & 5 & 2.7 & 7.74 \\
& 4 & 4 & 2.7 & 9.70 \\
& 5 & 10 & 2.7 & 7.75 \\
& 6 & 50 & 2.7 & 7.75 \\
\hline \hline
\end{tabular}


cases not shown in Fig. 7. It is seen that given the scatter in the experimental data, the accuracy of the predictions is not very sensitive to the constant values: nor is it sensitive to the functional forms examined.

This insensitivity does not undermine the need to use the momentum-accommodation function that switches from specular scattering to diffuse scattering as the particle radius increases. Without the use of the momentum-accommodation function, the TEM and mobility data cannot be reconciled over the entire range of particle size. This point is demonstrated by the dashed lines shown in Fig. 6, which depict the radius predicted in the limit of specular scattering and by the $90 \%$ diffuse and $10 \%$ specular mix.

The transition from specular to diffuse scattering happens at a particle radius of $2-3 \mathrm{~nm}$, based on the $\beta$ value [equal to 2.5 as shown in Eq. (20)] obtained by fitting the experimental data of Table III. This radius is larger than the value quoted by Tammet's [24], e.g., 1-2 nm in diameter. The switch function in Tammet's formula is a function of $A+B$ in the Stokes-Cunningham formula. Therefore, the smaller particle size for the transition may be attributed to the inaccuracy in the Stokes-Cunningham formula for nanosized particles. Regardless, the physical reason for this transition will have to be investigated in future studies.

\section{COMPARISON OF THE CURRENT THEORY WITH OTHER DATA}

Recently, Fernández de la Mora et al. [18] reported a comprehensive study of nanoparticle size determination by mobility analysis and focused impaction. The electric mobility values were measured as a function of particle size for a large number of silver particles with mass ranging from 3 to $160 \times 10^{-20} \mathrm{~g}$ or diameter ranging from about 1 to $4 \mathrm{~nm}$ (based on a mass density of $7.9 \mathrm{~g} / \mathrm{cm}^{3}[18]$ ). Furthermore, Kauffman et al. [25] reported the mobility of protein nanoparticles with mass ranging from 1 to $110 \times 10^{-20} \mathrm{~g}$ or diameter ranging from 1 to $6 \mathrm{~nm}$ (based on a mass density of $1.28 \mathrm{~g} / \mathrm{cm}^{3}$ [18]). These data provide the much needed test cases for the current theory.

A comparison between the aforementioned experiments and predictions from the current theory is made following the same fashion as that in [18]. The relevant potential function parameters and mass density values are given in Table IV. Figure 8 presents a $Z^{-1 / 2}$ versus $m_{p}^{1 / 3}$ plot, where the symbols are experimental data and lines are predicted from the current theory. Following the work of Fernández de la Mora et al., we used two different mass density values $\rho_{s}$ $=7.9$ and $10.5 \mathrm{~g} / \mathrm{cm}^{3}$ for silver particles. It is seen that the current theory agrees very well with the data for small particles using $\rho_{s}=7.9 \mathrm{~g} / \mathrm{cm}^{3}$ and for large particles with $\rho_{s}$ $=10.5 \mathrm{~g} / \mathrm{cm}^{3}$. Unlike the formula of Friedlander [8] and Fernández de la Mora et al. [18], which predict a linear dependence between $Z^{-1 / 2}$ and $m_{p}^{1 / 3}$, the current theory predicts a subtle curvature in the relation between $Z^{-1 / 2}$ and $m_{p}^{1 / 3}$. Obviously, the curvature is caused by the transition at around $2-3 \mathrm{~nm}$ in particle radius, from specular scattering for small particles to diffuse reflections for large particles.

The theory is found to be in good agreement with the

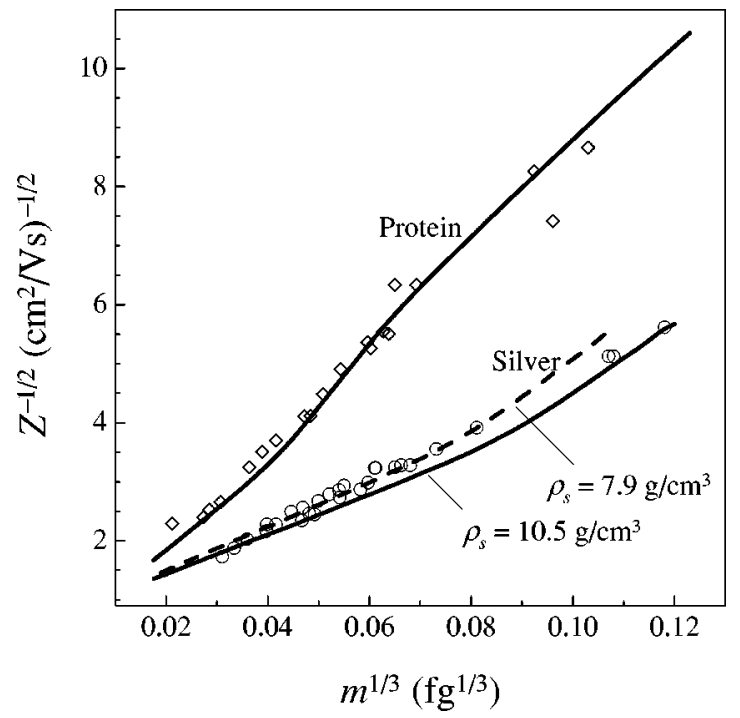

FIG. 8. Comparison of experiment (symbols) and theory (lines) for $Z^{-1 / 2}$ vs $m_{p}^{1 / 3}$. The silver data are taken from Fernández de la Mora et al. [18] and the corrected protein data taken from Kaufman et al. [25].

protein data. The minor discrepancy between experimental data and the current theory could be a consequence of the inaccuracy of the potential parameters used in our analysis. Since these parameters are not known, we opt to estimate them using the parameters of carbon (see Table IV). Obviously, these estimates are rough at best. The disagreement could also be caused by an inaccuracy in the momentumaccommodation function, as there is no theoretical evidence to confirm that this function is independent of the particle material and atomic structure on the particle surface. This issue will be examined in a future study.

\section{GENERALIZED DRAG-FORCE FORMULA}

The theoretical result of the free-molecule regime may be extended to the entire range of Knudsen number following a semiempirical approach, as outlined in the Appendix. Specifically, we propose that the drag force be modeled by

$$
\begin{aligned}
\mathbf{F} & =\frac{\alpha^{\prime}}{\left(1+\alpha^{\prime w}\right)^{1 / w}} \mathbf{F}_{\infty} \\
& =-\frac{8}{3} \frac{\alpha^{\prime}}{\left(1+\alpha^{\prime w}\right)^{1 / w}} \sqrt{2 \pi m_{r} k T} N R^{2} \Omega_{\text {avg }}^{(1,1)^{*}} \mathbf{V},
\end{aligned}
$$

where

$$
\alpha^{\prime}=\frac{9 \sqrt{2 \pi} \mu}{8 \Omega_{\text {avg }}^{(1,1) *} \sqrt{m_{r} k T} N R},
$$

and $w$ is an empirical constant obtained from a best fit to experimental data of Millikan [2,3]. The expression for $\alpha^{\prime}$ can be expressed equivalently in terms of the Knudsen number as 


$$
\alpha^{\prime}=\frac{45 \pi}{64} \sqrt{1+\frac{m_{g}}{m_{p}}} \frac{\mathrm{Kn}}{\Omega_{a v g}^{(1,1)^{*}} \Omega^{(2,2)^{*}}},
$$

where $\Omega^{(2,2) *}$ is the reduced collision integral of fluid viscosity [4]. Here it is worth noting that for $\mathrm{Kn} \ll 1$, we have $\alpha^{\prime} \rightarrow 0$. Putting Eq. (23a) into Eq. (22), we obtain $\mathbf{F}=$ $-6 \pi \mu R \mathbf{V}$, i.e., Stokes' drag-force equation under the stick boundary condition. For $\mathrm{Kn} \gg 1$, we have $\alpha^{\prime} \rightarrow \infty$, and therefore $\mathbf{F}=\mathbf{F}_{\infty}$.

Millikan [3] introduced the variable $A^{\prime}$, and in the context of the Stokes-Cunningham formula [Eq. (1)], he defined it as $A^{\prime}=A+B e^{-E / \mathrm{Kn}}$. The values of $A^{\prime}$ were indirectly measured for a large number of droplets $[2,3]$ and were later reanalyzed by Allen and Raabe [26]. We derived the dragforce values for these experiments using $F=6 \pi \mu R \mathrm{~V} /(1$ $+A^{\prime} \mathrm{Kn}$ ) and found that Eq. (22) with a $w$ value of 1.143 agreed well with these derived data. Here the viscosity of air is taken from Schilichting [30], i.e.,

$$
\mu=\mu_{0}\left(\frac{T}{293.15}\right)^{3 / 2}\left(\frac{293.15+T_{0}}{T+T_{0}}\right),
$$

where $\mu_{0}=1.819 \times 10^{-4} \mathrm{cp}$ and $T_{0}=110.4 \mathrm{~K}$.

Figure 9(a) shows the values of $F /(6 \pi \mu R V)$ derived from the $A^{\prime}$ data tabulated in [26] and those predicted by Eq. (22) [27], over a range of Knudsen number. It is seen that the agreement is very good. In Fig. 9(b), we plot the deviations of Eq. (22) in the predicted drag-force values and compare them to the Stokes-Cunningham formula (using the parameters of Allen and Raabe [26]). Clearly, the current theory is as accurate as the Stokes-Cunningham formula of the best known parameters over the entire range of Knudsen number. We note, therefore, that the ability to predict the mobility of nanosize particles and its rigorous theoretical foundation in the $\mathrm{Kn} \gg 1$ limit makes the current theory a far more general treatment for spherical particle transport in dilute gases than the Stokes-Cunningham formula.

\section{SUMMARY}

In the present paper we demonstrated a generalized treatment for the drag force, electric mobility, and diffusion coefficient of spherical particles in dilute gases and for $\mathrm{Kn} \gg 1$. The treatment is based on the theoretical derivation of par-

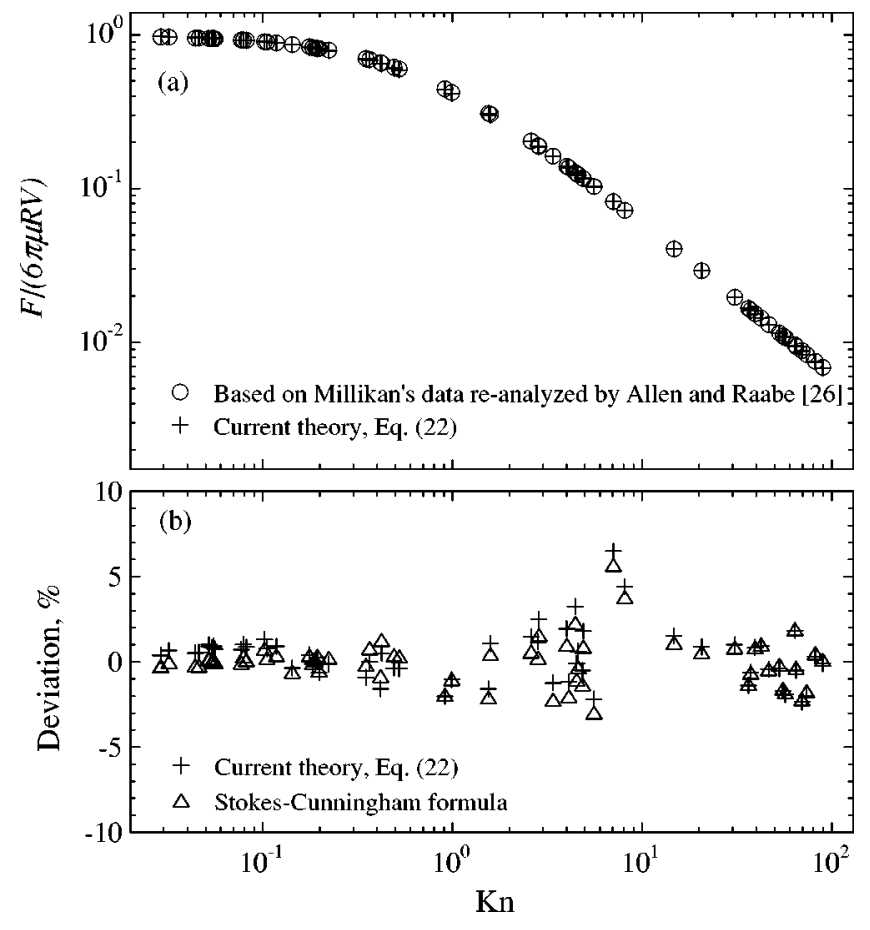

FIG. 9. (a) Comparison of $F /(6 \pi \mu R V)$ values based on the experimental data of Millikan [2,3] and those predicted using Eq. (22); (b) comparison of the percentage deviations of the drag force predicted from the Stokes-Cunningham formula $(A=1.155, B$ $=0.471$, and $E=0.596$ [26]) and from the current theory. The experimental $F /(6 \pi \mu R V)$ values were calculated from Millikan's $A^{\prime}=A+B e^{-E / \mathrm{Kn}}$ values given in [26].

ticle drag of paper I, obtained for two limiting scattering models. The current theory was validated against the available experimental data. Extending the current theory in the $\mathrm{Kn} \gg 1$ limit, we propose that the drag force for spherical particles in the entire range of the Knudsen number be treated by the following equation:

$$
\mathbf{F}=-\frac{8}{3}\left(1+\alpha^{\prime-1.143}\right)^{-0.875} \sqrt{2 \pi m_{r} k T} N R^{2} \Omega_{a v g}^{(1,1)^{*}} \mathbf{V},
$$

where the expression of $\alpha^{\prime}$ is given by Eq. (23b), the average collision integral $\Omega_{a v g}^{(1,1)^{*}}$ is given by

$$
\Omega_{\text {avg }}^{(1,1) *}=\frac{\Omega_{d}^{(1,1)^{*}}+\operatorname{Kn}\left(0.9 \Omega_{d}^{(1,1)^{*}}+0.1 \Omega_{s}^{(1,1)^{*}}\right)-0.9 \operatorname{Kn}\left(\Omega_{d}^{(1,1)^{*}}-\Omega_{s}^{(1,1)^{*}}\right) /\left[1+(R / 2.5)^{15}\right]}{1+\operatorname{Kn}},
$$

and last, the reduced collision integrals for diffuse and specular scattering are expressed by

$$
\begin{gathered}
\Omega_{d}^{(1,1)^{*}}=1+\frac{\pi}{8}+\left[1.072+\frac{2.078}{T^{* 1 / 4}}+\frac{1.261}{T^{* 1 / 2}}\right]\left(\frac{\sigma}{R}\right)+\left[3.285-\frac{8.872}{T^{* 1 / 4}}+\frac{5.225}{T^{* 1 / 2}}\right]\left(\frac{\sigma}{R}\right)^{2}, \\
\Omega_{s}^{(1,1)^{*}}=1+\left[0.316+\frac{1.47}{T^{* 1 / 4}}+\frac{0.476}{T^{* 1 / 2}}\right]\left(\frac{\sigma}{R}\right)+\left[1.53-\frac{5.013}{T^{* 1 / 4}}+\frac{4.025}{T^{* 1 / 2}}\right]\left(\frac{\sigma}{R}\right)^{2} .
\end{gathered}
$$


In the above equation, the modified reduced temperature is given by

$$
T^{*}=\frac{3 k T}{2} \frac{\bar{M}}{\pi \varepsilon \sigma^{3} \rho_{s}},
$$

and $\sigma=\left(\sigma_{g}+\sigma_{p}\right) / 2$ and $\varepsilon=\sqrt{\varepsilon_{g} \varepsilon_{p}}$, where $\sigma_{p}$ and $\varepsilon_{p}$ are the self-collision diameter and well depth of the Lennard-Jones potential function for the constituent atom or molecule of the particle material and $\sigma_{g}$ and $\varepsilon_{g}$ are those of the molecules of the gas media. Last, we note that Eq. (25) is generally applicable to other particle transport properties including the diffusion coefficient [5].

\section{ACKNOWLEDGMENT}

This work was supported by the National Science Foundation under Grant No. CHE-0089136.

\section{APPENDIX: A SEMIEMPIRICAL TREATMENT OF THE DRAG FORCE IN THE TRANSITION REGIME}

From the standpoint of continuum mechanics, the dragforce equations differ primarily in the boundary condition [28]. For the continuum regime, the stick boundary condition was assumed. At the microscopic level, this boundary condition implies that the relative velocity of the fluid molecules at the surface of the particle is zero. For the free-molecule regime, the particle exhibits no net effect on the velocity of the fluid molecules, which is referred to as the slip boundary condition. The transition regime represents an intermediate boundary condition, which will be treated here by the gaskinetic theory. Although the mathematical treatment below is rigorous, physically the approach is semiempirical. Because the transition regime corresponds to relatively large particles, we neglect the potential force of interactions between the particle and fluid molecule.

Consider a coordinate system shown in Fig. 10. The origin $O^{\prime}$ is an arbitrary point on the surface of the particle with $x$ axis being tangential to the surface and $z$ axis directing to the center of the particle. $\theta$ is the angle between the incoming fluid flow and the $z$ axis. Assuming the equilibrium velocity of gas molecules is denoted by $\mathbf{V}$ with $v_{x}, v_{y}$, and $v_{z}$ being the components in $x, y$, and $z$ directions, respectively. The velocity distribution function of the incident molecules, $f_{i}$, under the assumption of slip boundary condition, is given by [29]

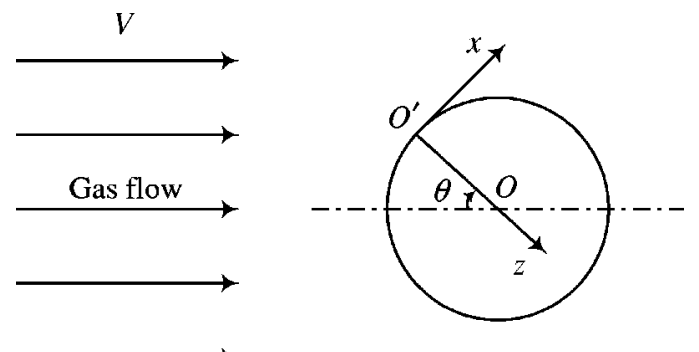

FIG. 10. Schematic of the coordinate system of free-molecule

$$
\begin{aligned}
f_{i}= & \frac{N}{\left(2 \pi k T / m_{r}\right)^{3 / 2}} \\
& \times \exp \left(-\frac{\left(v_{x}-V \sin \theta\right)^{2}+v_{y}^{2}+\left(v_{z}-V \cos \theta\right)^{2}}{2 k T / m_{r}}\right),
\end{aligned}
$$

and the velocity distribution function of the reflected molecules, $f_{r}$, is

$$
f_{r}=\frac{\psi}{2 \pi\left(k T / m_{r}\right)^{2}} \exp \left(-\frac{v_{x}^{2}+v_{y}^{2}+v_{z}^{2}}{2 k T / m_{r}}\right),
$$

where $\psi$ is the incident molecular flux,

$$
\psi=\int_{0}^{\infty} \int_{-\infty}^{\infty} \int_{-\infty}^{\infty} v_{z} f_{i} d v_{x} d v_{y} d v_{z} .
$$

For the transition regime, we assume that the overall effect of the particle on the velocity of gas molecules could be represented by a factor $\alpha^{*}$ and the velocity of the gas molecules around the particle is $\alpha^{*} V$ rather than $V$, so that Eq. (A1) is replaced by

$$
\begin{aligned}
f_{i}= & \frac{N}{\left(2 \pi k T / m_{r}\right)^{3 / 2}} \\
& \times \exp \left(-\frac{\left(v_{x}-\alpha^{*} V \sin \theta\right)^{2}+v_{y}^{2}+\left(v_{z}-\alpha^{*} V \cos \theta\right)^{2}}{2 k T / m_{r}}\right) .
\end{aligned}
$$

The incident molecular flux on the area element around the origin, $d A$, is

$$
n_{i}=\int_{0}^{\infty} \int_{-\infty}^{\infty} \int_{-\infty}^{\infty} v_{z} f_{i} d v_{x} d v_{y} d v_{z} d A,
$$

so that momentum flux is given by

$$
M_{i}=\int_{0}^{\infty} \int_{-\infty}^{\infty} \int_{-\infty}^{\infty} m_{r} V v_{z} f_{i} d v_{x} d v_{y} d v_{z} .
$$

The pressure and shear due to the incident molecules are the components of momentum flux of incident molecules in the $z$ and $x$ directions,

$$
\begin{gathered}
p_{i}=\int_{0}^{\infty} \int_{-\infty}^{\infty} \int_{-\infty}^{\infty} m_{r} v_{z}^{2} f_{i} d v_{x} d v_{y} d v_{z}, \\
\tau_{i}=\int_{0}^{\infty} \int_{-\infty}^{\infty} \int_{-\infty}^{\infty} m_{r} v_{x} v_{z} f_{i} d v_{x} d v_{y} d v_{z},
\end{gathered}
$$

respectively. Likewise, the pressure and shear due to the reflected molecules are

$$
p_{r}=\int_{-\infty}^{0} \int_{-\infty}^{\infty} \int_{-\infty}^{\infty} m_{r} v_{z}^{2} f_{r} d v_{x} d v_{y} d v_{z},
$$




$$
\tau_{r}=\int_{-\infty}^{0} \int_{-\infty}^{\infty} \int_{-\infty}^{\infty} m_{r} v_{x} v_{z} f_{r} d v_{x} d v_{y} d v_{z}
$$

The differential drag force $d F$ is therefore given by

$$
d F=\left[\left(p_{i}+p_{r}\right) \cos \theta+\left(\tau_{i}+\tau_{r}\right) \sin \theta\right] d A .
$$

Integrating Eq. (A11) over the surface of the particle, we obtain the drag force as

$$
\begin{aligned}
F= & 2 \pi R^{2} \int_{0}^{\pi}\left[\left(p_{i}+p_{r}\right) \cos \theta+\left(\tau_{i}+\tau_{r}\right) \sin \theta\right] \sin \theta d \theta \\
= & 2 \pi R^{2} N k T\left\{\frac{1+2 S^{2}}{2 S \sqrt{\pi}} \exp \left(-S^{2}\right)\right. \\
& \left.+\frac{S^{4}+S^{2}-1 / 4}{S^{2}} \operatorname{erf}(S)+\frac{\sqrt{\pi} S}{3}\right\},
\end{aligned}
$$

where $S=\alpha^{*} V / \sqrt{2 k T / m_{r}}$. In general, the value of $S$ is of the order of $10^{-3}$ if $\alpha^{*} V \sim 1 \mathrm{~m} / \mathrm{s}$. Expanding Eq. (A12) around $S=0$ in a Taylor series and truncating the terms higher than the second order, we obtain the first-order approximation for the drag force as

$$
F=\frac{8}{3}\left(1+\frac{\pi}{8}\right) \alpha * \sqrt{2 \pi m_{r} k T} N R^{2} V .
$$

In the above equation, the $(1+\pi / 8)$ term derives from the rigid-body collision assumption and can be replaced by the effective, reduced collision integral. Therefore, Eq. (A13) can be rewritten as

$$
F=\frac{8}{3} \Omega_{a v g}^{(1,1)^{*}} \alpha * \sqrt{2 \pi m_{r} k T} N R^{2} V .
$$

Obviously, $\alpha^{*}$ takes the limiting value of unity as $\mathrm{Kn} \rightarrow \infty$. On the other hand, the drag force should approach the limit of Stokes's equation as $\mathrm{Kn} \rightarrow 0$, i.e.,

$$
F_{0}=6 \pi \mu R V
$$

Equating Eqs. (A14) and (A15), we obtain the second limiting $\alpha^{*}$ value as

$$
\alpha^{*} \rightarrow \alpha^{\prime}=\frac{9 \sqrt{2 \pi} \mu}{8 \Omega_{a v g}^{(1,1)^{*}} \sqrt{m_{r} k T} N R} .
$$

We expect that in the transition regime the velocity of gas molecules lies between $V$ and $\alpha^{\prime} V$. For this reason, we introduce an empirical expression to model the transition regime as

$$
\alpha^{*}=\frac{\alpha^{\prime}}{\left(1+\alpha^{\prime w}\right)^{1 / w}},
$$

where $w$ is an empirical constant. It can be shown that for $\mathrm{Kn} \rightarrow 0$, we have $\alpha^{\prime} \ll 1$, and therefore $\alpha^{*}=\alpha^{\prime}$. With $\mathrm{Kn} \rightarrow \infty$, we have $\alpha^{\prime} \gg 1$ and $\alpha^{*}=1$. The drag force over the entire range of Knudsen number is then modeled by

$$
F=\alpha^{*} F_{\infty}
$$

Of course, the above equation satisfies the two limiting boundary conditions in the large- and small-Knudsennumber limits.
[1] R. A. Millikan, Science 32, 436 (1910).

[2] R. A. Millikan, Philos. Mag. 34, 1 (1917).

[3] R. A. Millikan, Philos. Mag. 22, 1 (1923).

[4] Z. Li and H. Wang, preceding paper, Phys. Rev. E 68, 061206 (2003).

[5] The drag force $\mathbf{F}$, diffusion coefficient $D$, and electric mobility in the limit of zero field $Z$ are bound by the same drag coefficient, $k_{d}$, viz., $\mathbf{F}=-k_{d} \mathbf{V}, D=k T / k_{d}$, and $Z=q / k_{d}$, where $\mathbf{V}$ is the relative velocity vector of a particle moving in a fluid, $q$ is the charge in the particle, $k$ is the Boltzmann constant, and $T$ is the temperature. Therefore, $k_{d}, D$, and $Z$ are equivalent attributes of the transport property of a small particle.

[6] S. Chapman and T. G. Cowling, The Mathematical Theory of Non-Uniform Gases (Cambridge University Press, Cambridge, England, 1970).

[7] J. O. Hirschfelder, C. F. Curtiss, and R. B. Bird, Molecular Theory of Gases and Liquids (Wiley, New York, 1954).

[8] S. K. Friedlander, Dust, Smoke and Haze (Wiley, New York, 1977).

[9] P. S. Epstein, Phys. Rev. 23, 710 (1924).

[10] V. Ya. Rudyak and S. L. Krasnolutski, in Proceedings of the XXI International Symposium on Rarefied Gas Dynamics, Marseille, 1999, edited by L.J.F. Hermans, B. Nagels, and P.
Bakker (Cepadues-Editions, Toulouse, France, 1998), Vol. 1, p. 263.

[11] V. Ya. Rudyak and S. L. Krasnolutski, Dokl. Phys. 46, 897 (2001).

[12] V. Ya. Rudyak and S. L. Krasnolutski, Dokl. Phys. 47, 758 (2002).

[13] C. Farina, F. C. Santos, and A. C. Tort, Am. J. Phys. 67, 344 (1999).

[14] T. R. Marrero and E. A. Mason, J. Phys. Chem. Ref. Data 1, 1 (1972).

[15] A. Boushehri, J. Bzowski, J. Kestin, and E. A. Mason, J. Phys. Chem. Ref. Data 16, 445 (1987).

[16] D. Timple, K. Scheerschmidt, and S. H. Garofalini, J. NonCryst. Solids 221, 187 (1997).

[17] D. R. Lide, CRC Handbook of Chemistry and Physics, 84th ed. (CRC Press, Boca Raton, FL, 2003).

[18] J. Fernández de la Mora, L. de Juan, K. Liedtke, and A. Schimidt-Ott, J. Aerosol Sci. 34, 79 (2003).

[19] H. Tammet, J. Aerosol Sci. 3, 459 (1995).

[20] H. G. Scheibel and J. Porstendörfer, J. Aerosol Sci. 14, 113 (1983).

[21] Y. Kuga, K. Okauchi, D. Takeda, Y. Ohira, and K. Ando, J. Nanopart. Res. 3, 175 (2001).

[22] The viscosity value list in [17] is $1.8 \times 10^{-4} \mathrm{cp}$ for at $300 \mathrm{~K}$. The $1.78 \times 10^{-4} \mathrm{cp}$ value used in the current analysis was ob- 
tained by a simple temperature correction given by 1.8 $\times 10^{-4}(294 / 300)^{1 / 2}$.

[23] U. S. Schwartz, S. A. Safran, and S. Komura, in Dynamics in Small Confining Systems V, edited by J.M. Drake, J. Klafter, P. Levitz, R.M. Overney, and M. Urbakh, Mater. Res. Soc. Symp. Proc. No. 651 (Material Research Society, Boston, 2001), p. T5.3.1.

[24] H. Tammet, J. Aerosol Sci. 26, 459 (1995).

[25] S. L. Kaufman, J. W. Skogen, F. G. Dorman, F. Zarrin, and L. C. Lewis, Anal. Chem. 68, 1895 (1996); 68, 3703 (1996).
[26] M. D. Allen and O. G. Raabe, J. Aerosol Sci. 13, 537 (1982).

[27] Only 64 out of a total of 75 oil droplets in Millikan's work are used for the comparison. The measurements of the rest oil droplets might involve some error (see Allen and Raabe [26]).

[28] G. G. Stokes, Proc. Cambridge Philos. Soc. 9, 8 (1851).

[29] E. H. Kennard, Kinetic Theory of Gases (McGraw-Hill, New York, 1938).

[30] H. Schilichting, Boundary Layer Theory (McGraw-Hill, New York, 1968). 